\title{
Soziale Kontrolle. Begriff - Probleme beim Umgang mit ihm - Seine Aktualität
}

\author{
Helge Peters
}

Eingegangen: 4. Mai 2020 / Angenommen: 11. August 2020 / Online publiziert: 5. November 2020

(C) Der/die Autor(en) 2020

Zusammenfassung Zunächst geht es um den Begriff „,soziale Kontrolle“. Sie wird als Handeln verstanden, das auf den Erhalt von Normkonformität in dem Bereich zielt, in dem es ausgeübt wird. Es zielt nicht einfach auf die Konformität ihrer AdressatInnen. Sodann wird die Rezeption des Begriffs ,,soziale Kontrolle“ in neueren devianz-, kontroll- und herrschaftssoziologischen Texten dargestellt. Gezeigt werden Widersprüchlichkeiten und Tendenzen, nach denen Maßnahmen als soziale Kontrolle beschrieben werden, die auf den Machterhalt ihrer Akteure, nicht jedoch auf den Erhalt von Normkonformität zielen. Problematisiert werden auch die empirischen Grundlagen der sog. Punitivitätsthese. Schließlich wird das Gewicht erörtert, das die gegenwärtige deutsche Soziologie dem Begriff „,soziale Kontrolle“ beimisst. Es ergibt sich, dass der Begriff an soziologischer Relevanz eingebüßt hat. Dies wird bedauert. Mit dieser Einbuße könne auch die soziologische Aufmerksamkeit für das mögliche Verfehlen der Kontrollziele und für Devianzkonstrukte schwinden, die von Instanzen sozialer Kontrolle hergestellt werden.

H. Peters $(\bowtie)$

Institut für Sozialwissenschaften, Carl von Ossietzky Universität Oldenburg, Ammerländer

Heerstraße 114-118, Oldenburg, Deutschland

E-Mail: helge.peters@uni-oldenburg.de 


\title{
Social Control. Term-Problems Dealing With It-Its Present Relevance
}

\begin{abstract}
At first, this essay deals with the term of "social control". It is understood as an action that aims to the conformity of compliance with the standards in that social field where it is practised. It does not simply aim at the conformity of its addressees. Then, the reception of the term "social control" in recently published deviance-, control- and governmenttheoretical texts is described. Contradictions and tendencies are pointed out according to which measures are described as social control which actually aim at support of power of the protagonists and not at conformity to standards. Moreover, the empirical bases of the so-called thesis of punitivity are called into question. Finally, the importance is discussed which the contemporary German sociology attaches to the term of "social control". It is demonstrated that this importance has diminished. The author regrets this development. Because it was to be feared that this reduction also would diminish the attention which is paid for the possibility of reducing the achievement of goals of social control. Moreover, it was to be feared that the sociological attention for the constructs of deviance which are produced by agencies of social control could be reduced as well.
\end{abstract}

\section{Begriff}

Soziale Kontrolle ist Handeln. Es ist ein menschliches Verhalten, mit dem - so sagt Max Weber - der oder die Handelnden einen subjektiven Sinn verbinden (vgl. 1964, S. 3). Arten des Handelns sind demzufolge nach dem subjektiven Sinn zu unterscheiden, den die Handelnden ihrem Handeln geben. Ginge es Akteuren sozialer Kontrolle bei ihrem Handeln um die Herstellung von Normkonformität ihrer AdressatInnen wäre dies der subjektive Sinn ihres Handelns.

Empfiehlt es sich, bei der Bestimmung des Begriffs „,soziale Kontrolle“ Weber zu folgen? Es gibt Gegenpositionen. So bezeichnet Doris Mathilde Lucke in ihrem Versuch, den Begriff „soziale Kontrolle“ zu definieren, dieses Handeln als einen „Mechanismus der Integration von Gesellschaften“, der „,der Aufrechterhaltung sozialer Ordnung“ diene. Weiter schreibt Lucke, über soziale Kontrolle werde ,der Zerfall von Gesellschaften verhindert" (2014, S. 245; Hervorhebungen im Original).

Was heißt „dienen“, was heißt ,verhindern“? Die Annahmen, dass ein Handeln einer Sache - einem Zweck, einem Ziel - dient oder dass ein Handeln etwas verhindert, besagt, dass dieses Handeln etwas bewirkt. Wäre also das Urteil eines/er StrafrichterIn, das Empörung bewirkt, zu verbreiteten Protesten führt, also nicht besagter Ordnung dient, am Ende gar den „Zerfall“ der Gesellschaft bewirkt, keine soziale Kontrolle? Variiert der Charakter einer Handlung mit ihren Wirkungen? Ist das skizzierte Urteil je nach Wirkung einmal soziale Kontrolle und einmal nicht?

Macht man die Handlungswirkungen zum Definitionsmerkmal des Begriffs ,,soziale Kontrolle“, so würde er also unscharf. Dies spricht zunächst einmal dafür, soziale Kontrolle im Sinne Webers als ein Verhalten mit subjektivem Sinn zu verstehen oder alltagssprachlich: die Handlungsabsicht zum Merkmal der Definition des 
Begriffs „soziale Kontrolle“ zu machen. Von ihr wäre als von einem Vorgang zu reden, der auf die Herstellung von Normkonformität zielt.

Damit wäre der Begriff allerdings immer noch recht unscharf. Einer seiner Mängel wird z.B. in dem Versuch von Tobias Singelnstein und Peer Stolle deutlich, soziale Kontrolle zu definieren. In ihrem viel gelesenen Buch „Die Sicherheitsgesellschaft“ schreiben sie: Sozialkontrolle reiche ,,von der Sozialisation als Vermittlungsinstanz über Familie, Schule und soziales Umfeld bis hin zur staatlichen Strafverfolgung und umfasst dementsprechend sehr unterschiedliche Institutionen, Mechanismen und Techniken“ (2012, S. 11). Zu fragen ist: Was unterscheidet dann etwa die Begriffe „Sozialisation“, „Erziehung“, ,soziale Zwänge“ oder „Institutionalisierung“ von dem Begriff „,soziale Kontrolle“? Er wäre überflüssig. Derselbe Einwand trifft auch den Begriff ,soziale Kontrolle“, den Steffen Mau in seinem - ebenfalls viel gelesenen - Buch „Das metrische Wir“ (2017) verwendet. Es geht in dieser Schrift um die „Quantifizierung des Sozialen“ (Untertitel). Diese Quantifizierung habe so der Klappentext - ,das Potenzial, ein neues Regime der Ungleichheit hervorzubringen, in dem wir immerfort bewertet sowie mit anderen verglichen werden“. Mau beschreibt z.B. die gegenwärtig verbreitete Neigung vieler Menschen, sich öffentlich - z. B. beim Dauerlaufen - miteinander zu messen. Er versucht, diese Neigung mit der Annahme zu erklären, ,dass schon die Präsenz von Beobachtern Leistungssteigerungen auslösen kann“ (2017, S. 180). Verbunden seien derartige Aktivitäten mit freiwilliger Datenerhebung. So entstehe ,ein Netzwerk wechselseitiger Sozialkontrolle, das die ,richtigen “ Verhaltensweisen bestärkt und die ,falschen “ zurückdrängt“ (ebd.). Was Mau hier beschreibt, sind - so lautet die Abschnittsüberschrift - „Motivationstechniken“ (2017, S. 178), d. h. Techniken, die der Selbst- und Fremdsteuerung dienen. Es handelt sich also um Vorgänge, die als Sozialisation, Erziehung, soziale Zwänge, Selbstzwänge, vielleicht auch als Sozialdisziplinierung bezeichnet werden könnten. Der Begriff ,,soziale Kontrolle“ brächte hier keine neuen Einsichten. Die aufgeführten Begriffe bezeichnen ziemlich klar, was hier gemeint ist. An anderer Stelle spricht Mau von der mit der Verbreitung der Soziometrie verbundenen „Utopie vollständiger Transparenz“ und der „Dystopie totaler Kontrolle“ (2017, S. 231). Beide seien Ausdruck der Leidenschaften des Quantifizierens. Die neue Informationsflut lasse unser soziales Leben nicht nur immer gläserner werden. Das unermüdliche Datensammeln kippe auch um in eine Tyrannei der ,exzessiven sozialen Kontrolle“ (ebd.). Viele Quantifizierungspraktiken übernähmen Aufgaben der Steuerung sowie der Belohnung. Dergleichen konditioniere uns auf bestimmte Ziele (vgl. ebd.).

Wiederum: Das sind Vorgänge der Sozialisation, der Institutionalisierung, der Sozialdisziplinierung, der sozialen Zwänge. Des Begriffs ,,soziale Kontrolle“ bedarf es hier nicht.

Um dem in solchen Erörterungen deutlich werdenden Mangel an Schärfe und Spezifizität des Begriffs ,soziale Kontrolle“ abzuhelfen und ihm wieder die Berechtigung einer eigenständigen soziologischen Existenz zu geben, empfiehlt es sich immer noch, auf den Gedanken zurückzukommen, den Peter L. Berger und Thomas Luckmann angesichts des auch von ihnen festgestellten unspezifischen Gebrauchs des Begriffs „,soziale Kontrolle“ formulieren. „Die primäre soziale Kontrolle ergibt sich“, so schreiben sie, „durch die Existenz von Institutionen überhaupt. Wenn ein 
Bereich menschlicher Tätigkeit institutionalisiert wird, so bedeutet das eo ipso, daß er unter sozialer Kontrolle steht" (1969, S. 59). Berger und Luckmann übernehmen damit zunächst einmal den weiten und unpräzisen Kontrollbegriff, den wir bemängeln. Dann aber fahren sie fort: „Zusätzliche Kontrollmaßnahmen sind nur erforderlich, sofern Institutionalisierungsvorgänge zu ihrem eigenen Erfolg nicht ganz ausreichen“ (ebd.). Diese zusätzlichen Kontrollmaßnahmen sind Berger und Luckmann zufolge Reaktionen auf gegenwärtiges oder erwartetes abweichendes Verhalten, die erfolgen, um dieses künftig zu verhindern (vgl. ebd.).

Hinreichend genau definiert ist damit der Begriff „,soziale Kontrolle“ aber immer noch nicht. Zu fragen wäre auch hier: Was heißt ,,verhindern“? Verhindern heißt nicht unbedingt, die Konformität der KontrolladressatInnen wiederherstellen zu wollen. Auch Maßnahmen des Ausschließens, der Verbannung und Tötung sind Maßnahmen sozialer Kontrolle. Sie zielen auf den Erhalt von Normkonformität in dem Bereich, in dem sie ergriffen werden.

Bei dem jetzigen Stand unserer Überlegungen können wir sagen: Wir haben zwei Probleme, die die soziologische Brauchbarkeit des Begriffs ,,soziale Kontrolle“ beeinträchtigen, mit der Festlegung der Handlungsabsicht als Definitionsmerkmal und mit der an Berger und Luckmann orientierten Engfassung des Begriffs gelöst. Zu fragen ist allerdings auch jetzt noch, ob ein so gefasster Begriff „,soziale Kontrolle“ der soziologischen Praxis genügen kann. Zu erörtern wäre zunächst die Rede Bergers und Luckmanns: ,,... sofern die Institutionalisierungsvorgänge selbst zu ihrem Erfolg nicht ganz ausreichen“. Man merkt dieser Formulierung an, dass Berger und Luckmann in ihrem Buch, obwohl es den soziologischen Konstruktivismus mitbegründet oder doch stark zu seiner Verbreitung beigetragen hat, abweichendes Verhalten noch nicht unter konstruktivistischer Perspektive betrachtet haben. Angenommen wird mit der zitierten Formulierung, Gesellschaft sei ein kulturhomogenes Gebilde. Verkannt wird damit, dass AdressatInnen sozialer Kontrolle oft nichts mit der von den VertreterInnen der Instanzen sozialer Kontrolle repräsentierten Kultur zu tun haben. Die Institutionalisierungsvorgänge haben danach nicht ,nicht ganz ausgereicht“. Sie sind vielmehr in Richtungen gegangen, die den AdressatInnen sozialer Kontrolle gänzlich fremd sind. Der Charakter als Vorgang der Durchsetzung herrschender Kultur gerät mit Bergers und Luckmanns Begriff aus dem Blick.

Als problematisch gilt seit der konstruktivistischen Wende der Soziologie abweichenden Verhaltens auch Bergers und Luckmanns Vorstellung, dass soziale Kontrolle auf Erfolgsmängel reagiere. Noch im Rahmen herkömmlichen ätiologischen Denkens bleibt der mit der Karrierethese begründete Einwand, dass der Zugriff von Instanzen sozialer Kontrolle Devianz schafft. Dieser Zugriff verringere die sozialen Teilnahmechancen der AdressatInnen, disponiere sie deswegen zur - wie Edwin M. Lemert (1951) es ausgedrückt hat - ,,sekundären“ Devianz. Angenommen wird hier die Existenz eines reaktionsbegründenden ,,kleinen“ objektiv vorliegenden Anlasses, die Existenz ,primärer“ Devianz. Soziale Kontrolle ist danach keine Reaktion auf ,sekundäre“ Devianz. Diese ist vielmehr Reaktion auf jene.

Weiter geht der konstruktivistische Einwand. Danach liegt der Kontrollgegenstand objektiv nicht vor. Er wird von KontrolleurInnen durch kontextorientierte Zuschreibung und Thematisierung geschaffen. 
Aber wären das Einwände gegen Bergers und Luckmanns Kontrollbegriff? Aus handlungstheoretischer Sicht könnte man sagen: Die KontrolleurInnen verstehen ihre Handlungen als Reaktionen auf Devianz. Dem subjektiven Sinn ihrer Handlungen liegt folgende Definition der Situation zugrunde: „Devianz liegt objektiv vor. Wir reagieren in der Absicht, Devianz künftig zu verhindern. " StrafrichterInnen unterstellen neueren Untersuchungen zufolge solche Situationsdefinitionen. Es gehe in Strafverfahren um die ,amtswegige Suche nach dem ,wahren Sachverhalt “", der - wie Charlotte Schmitt-Leonardy, Klaus Volk zitierend, schreibt - im Wege einer umfassenden Beweisaufnahme wie ein ,,vergrabenes Goldstück“ gefunden werden könne (2019, S. 214). Subjektiv definieren oder konstruieren RichterInnen also nicht. Sie „finden“ in der Absicht, soziale Kontrolle auszuüben. Die soziologische Brauchbarkeit des mit Bergers und Luckmanns Annahmen begründeten Begriffs soziale Kontrolle wird also mit der konstruktivistischen Kritik nicht rundweg bestritten. Er beschreibt wahrscheinlich großenteils das Selbstverständnis der KontrolleurInnen und damit den subjektiven Sinn ihres Handelns. Der soziologische Mangel dieses Begriffs besteht darin, dass er nicht anzuschließen ist an die neuere konstruktivistische Diskussion. Seine eigene Konstruktivität wird verkannt. Das mindert seine für eine soziologisch brauchbare Definition des Begriffs ,,soziale Kontrolle“ erforderliche Qualität jedoch nicht.

\section{Probleme beim Umgang mit dem Begriff ,soziale Kontrolle“}

2.1 Auch bei diesem Stand unserer Überlegungen lässt der Begriff ,,soziale Kontrolle" noch viele Fragen offen. $\mathrm{Zu}$ bedenken sind beispielsweise die Reichweite der Maßnahmen sozialer Kontrolle, die „Legitimität“ der Instanzen sozialer Kontrolle, deren Durchsetzungschancen usw. Es gibt hinreichend Literatur, in der diese Fragen beantwortet werden (vgl. etwa Peters 2002, S. $115 \mathrm{ff}$., 2009, S. 136ff., 2012, S. 1261; Menzel 2019, S. 73 f.). Wir müssen die in dieser Literatur vorgetragenen Erörterungen hier nicht wiederholen. Stattdessen sollen uns im Folgenden einige Probleme interessieren, die der soziologische Umgang mit dem Kontrollthema mit sich gebracht hat.

Als wichtige Instanzen sozialer Kontrolle im Sinne unseres Begriffs gelten die Strafjustiz, die Polizei, die Sozialarbeit, die Psychiatrie, aber auch - vor allem strafrechtliche - Sanktionsdrohungen, präventive Sicherheitsmaßnahmen wie Teile der Sozialpolitik und der Einsatz von Techniken der Selbstführung. Mit Beginn des 21. Jahrhunderts verbreiten sich in der Soziologie sozialer Kontrolle Annahmen, denen zufolge diese Instanzen sozialer Kontrolle ihre Strategien der Devianzbekämpfung seit den 1970/80er Jahre verändert hätten. Zu nennen ist hier an erster Stelle das Buch „The Culture of Control“ von David Garland (2001). Diese Arbeit hat die sozialwissenschaftliche Diskussion sozialer Kontrolle in den vergangenen Jahrzehnten stark beeinflusst. Garland nimmt an, dass die bis in die 1970er-Jahre in den ,westlichen“ Staaten verbreitete wohlfahrtsstaatliche Bearbeitung von Devianz - der ,penal welfarism“, wie es bei Garland heißt - in Misskredit geraten ist. Die Zeit des wirtschaftlichen Aufschwungs sei zu Ende gewesen. Ölkrise und beginnende Massenarbeitslosigkeit hätten gesellschaftliche Spannungen entstehen lassen. Die 
Kriminalität sei gestiegen. Dies alles habe zum Ende des „penal welfarism“ geführt. Abgelöst worden sei diese Politik durch zwei Strategien: durch die Strategie der ,präventiven Partnerschaft“ und durch die Strategie der ,punitiven Segregation“.

Der zuerst genannten Strategie entspreche die Kooperation öffentlicher und privater Instanzen bei der Bearbeitung von Kriminalität. Es gehe nicht mehr um Abschreckung und Bekämpfung. Ziel sei es vielmehr, das für unlösbar gehaltene Problem Kriminalität zu managen. Die Gelegenheiten für kriminelles Handeln müssten verringert, das Bewusstsein der Verbreitung von Kriminalität müsse geschärft, die Gesellschaft müsse so organisiert werden, dass sich die Neigung zur Kriminalität vermeidenden Selbststeuerung verbreite (vgl. Garland 2001, S. 140 f.; Singelnstein und Stolle 2012, S. 56 ff..). Politisch-pragmatischer Hintergrund dieser Strategie sei der Neoliberalismus mit seiner Hochschätzung individueller Verantwortung.

Die an zweiter Stelle genannte Strategie setzt dagegen auf Dramatisierung des Kriminalitätsproblems. Daneben gehe es aber auch um den Schutz vor Kriminalität. Diese Strategie sei populistisch und diene politischen Zwecken. Ihr entsprächen Parolen wie „Three strikes and you are out“. Eine wichtige Rolle spiele das Kriminalitätsopfer. Dessen Schicksal werde im Interesse populistischer Kriminalpolitik dramatisiert (vgl. Garland 2001, S. 142ff..). Politisch pragmatischer Hintergrund dieser Strategie sei der religiös-moralische Konservativismus.

Die Annahme der Koexistenz beider Strategien ist großenteils als nur scheinbar widersprüchlich wahrgenommen worden. Die dieser Annahme zugrundeliegenden Ideologien widersprächen einander zwar in ihren Grundaussagen. Für die ideologische Legitimierung gegenwärtiger Sozialkontrolle böten sie aber jeweils wichtige, sich ergänzende Bausteine, schreiben Singelnstein und Stolle (vgl. 2012, S. 46). Der Neoliberalismus stelle nur eine Position mit einer eigenen Werte- und Moralskala dar. Er könne gut mit einem autoritär-diktatorischen Regime kooperieren (vgl. Singelnstein und Stolle 2012, S. 52f.; vgl. auch Schlepper 2014, S. 193 ff.).

Eine Soziologie sozialer Kontrolle können solche Harmonisierungsbemühungen nicht recht befriedigen. Es mag sein, dass Regierungen beide Strategien einsetzen. Aber man wüsste schon gern, unter welchen sozialen Umständen sich welche Regierung für welche Strategie entscheidet. Garlands Buch suggeriert ein wenig, dass der Einsatz der Strategien mit der „Schwere“ der Kriminalität variiert. So schreibt der Autor z.B., dass die Fälle, die im Rahmen der an zweiter Stelle genannten Strategie bearbeitet würden, als normale Aspekte moderner Gesellschaften wahrgenommen würden (vgl. 2001, S. 128). Aber es geht nicht um die Differenz, die zwischen „schwer“ und ,leicht“ besteht - und unter konstruktivistischen Gesichtspunkten auch nicht bestehen kann. Als „,schwer“ oder „leicht“ werden Fälle ja erst definiert. Ihre „Schwere“ liegt nicht in ihrer Natur. Wenn SoziologInnen meinen, die Kriminalitätsbearbeitung mit der ,punitive segregation“-These, etwa mit der „Governing through Crime“"-Annahme, erklären zu können, können sie nicht gleichzeitig annehmen, Kriminalität werde gewissermaßen nur technisch bearbeitet.

$\mathrm{Zu}$ fragen wäre doch: Warum nutzen Regierungen einmal die Chancen, die ihnen die Dramatisierung von Kriminalität bietet, und einmal nicht? Es ist hier also ein Mangel der Garlandschen Konzeption moderner sozialer Kontrolle festzustellen. Es bleibt unklar, weshalb das eine „Baustein“ für das andere sein sollte. Garland beschreibt übrigens selbst - wahrscheinlich ungewollt - diesen Mangel mit dem 
Satz: „But government authorities ... are deeply ambivalent about these strategies, and they frequently retreat from their implications" (2001, S. 140). „Ambivalent"? Das heißt ja meist: Mit den uns verfügbaren Annahmen kommen wir einer Erklärung nicht näher.

Und dieser Mangel verweist auf ein weiteres Problem der Soziologie sozialer Kontrolle Garlands. Im Grunde wird mit der Annahme der Koexistenz beider Strategien eine der zentralen Thesen Garlands in Frage gestellt: die mit der Beschreibung der ,punitive segregation“ angenommene Tendenz zu einer punitiven Wende sozialer Kontrolle. Fritz Sacks Rede ist zuzustimmen, nach der die von uns skizzierte, von ihm als „dualistische Typologie“ bezeichnete Dualität „ganz offensichtlich quer zu der generellen These der punitiven Wende" liegt (Sack 2008, S. 308). Die von Sack als „Typ der De-Dramatisierung“ bezeichnete, von uns an zweiter Stelle genannte Strategie passe zur Wende der Kriminalpolitik so gar nicht (vgl. Sack 2008, S. 308).

2.2 Wir haben das Buch Garlands ohne Vorbehalte als eine Arbeit vorgestellt, deren Inhalt als Beitrag für eine Soziologie sozialer Kontrolle gelten kann. Das ist nicht ganz selbstverständlich. Richtig ist, dass mit der „strategy of preventive partnership“ soziale Kontrolle im Sinne unseres Begriffs beschrieben wird. Es geht im Wesentlichen darum, die weitere Verbreitung von Kriminalität zu verhindern. Auch die mit der ,strategy of punitive segregation“ verbundene soziale Kontrolle soll die Verbreitung von Kriminalität verhindern. Dies aber ist nur ein Ziel dieser Strategie. Es geht auch um populistische Maßnahmen des Herrschaftserhalts. Die im Zuge des Einsatzes dieser Strategie erfolgenden Kriminalitätsdramatisierungen zielen nicht auf die Konformität (potenzieller) Krimineller.

Garland geht hier von einer Annahme aus, die in der Literatur zur Kontrollthematik verbreitet ist: Kriminalität gehört danach - in den Worten von Murray Edelmans - zu den „bedauerlichen Verhältnissen, die günstige Gelegenheiten schaffen“ (1988, S. 177). Zu nennen sind hier neben vielen anderen Nils Christie und Jonathan Simon. „Günstig“ ist das „,bedauerliche Verhältnis“ Kriminalität, weil deren Bearbeitung Herrschaft nützen kann.

Christie glaubt, dass PolitikerInnen zu beweisen trachteten, im Kampf gegen Kriminalität führend zu sein. Die Politik sei eine Arena geworden, in der sich Individuen und Parteien gegenseitig mit der Forderung nach harten Maßnahmen zu übertreffen versuchten. Ursache für diese Konkurrenz sei der Kompetenzverlust des Staats. Dieser habe sich aus der Sozialpolitik zurückgezogen. Es gäbe nur noch wenige Gebiete, auf denen sich Politiker profilieren könnten. Die Kriminalitätsbekämpfung sei eines dieser Gebiete. Der Kampf gegen das Verbrechen werde unentbehrlich zur Schaffung der Legitimität in dem und für den ausreichend geschwächten Staat (vgl. 2005, S. 57 ff.). Behauptet wird hier also das „Governing through Crime“, nicht das „Governing by Law“. In verschiedenen Arbeiten vertritt auch Simon diese These. Er erläutert sie anhand von Forderungen konservativer PolitikerInnen der USA, die Todesstrafe zu erhalten oder einzuführen. Diese Strafe sei eine Quelle der öffentlichen Unterstützung der Staatsmacht geworden. Ihre Thematisierung gehe einher mit einer Thematisierung der Gewalt. „Gewalt“ werde insbesondere von „einfachen Leuten" gern erörtert. Sie versprächen sich davon eine Absicherung ihres von ihnen bedroht geglaubten Status' (vgl. 1997, S. 284). 
Offensichtlich wird in den Ausführungen Christies und Simons etwas als soziale Kontrolle im Sinne unseres Begriffs ausgegeben, was keine soziale Kontrolle in diesem Sinne ist. Fraglich ist, ob auch die viel zitierte Arbeit „Bestrafen der Armen“ von Loic J. D. Wacquant (2009) soziologischen Arbeiten dieser Art zuzurechnen ist. Wacquant sieht in den Strafrechts- und Strafverfolgungspraktiken der USA und des UK den Versuch, neoliberale Staatskunst zu verwirklichen. Deren wesentliches Element sei die Hochschätzung des Markts. Er gelte als Mechanismus, der nahezu alle menschlichen Interaktionen am besten organisieren könne. Ein Ausdruck dieser Hochschätzung sei die Deregulierung wirtschaftlicher Vorgänge und damit der Abbau des Sozialstaats. Dies führe zu sozialen Erschütterungen. Dem träten Regierungen mit der an die Betroffenen gerichteten Forderung entgegen, auch dürftigste Jobs anzunehmen. Maßnahmen, die dieser Forderung folgten, bezeichnet Wacquant als ,workfare“. Entsprächen die Adressaten dieser Forderung nicht, würde ihnen der Prozess mit der häufigen Folge der Inhaftierung gemacht. Maßnahmen dieser Art bezeichnet Wacquant als ,prisonfare“. Die Strafrechtspolitik breche also mit dem „Kriminalitäts-Strafe-Modell“. Die Politik ziele vielmehr darauf, deren Adressaten durch ,workfare“ in Schach zu halten oder durch ,prisonfare“ unsichtbar zu machen (vgl. Wacquant 2009, S. $88 \mathrm{ff} . ., 306 \mathrm{ff}$.).

Die Vorbehalte, diese Arbeit Wacquants als eine Variante der zuvor skizzierten sozialwissenschaftlichen Thesen zu bezeichnen, ergeben sich aus der Ausdeutbarkeit des „Unsichtbarmachens“. Dies könnte man als Fall sozialer Kontrolle verstehen, die ja auch darin bestehen kann, Adressaten auszuschließen. Wäre ein Adressat „unsichtbar gemacht“ worden, wäre das wohl gleichbedeutend mit ,,ausgeschlossen“. Damit aber wäre wohl nicht recht das getroffen, was Wacquant meint. Ihm zufolge geht es beim „Unsichtbarmachen“ nicht um das Verhindern von Devianz in dem Bereich, in dem Instanzen sozialer Kontrolle operieren. Vielmehr ziele die Strafrechtspolitik auf die Spaltung der Gesellschaft. Der Strafverfolgungsapparat helfe dem Staat ,durch selektive Durchdringung des sozialen und physikalischen Raums Kategorien durchzusetzen, materielle und symbolische Spaltungen aufrechtzuerhalten und Beziehungen und Verhalten zu prägen“, schreibt Wacquant (2009, S. 307). Er nimmt also an, dass es der Strafrechts- und der Strafverfolgungspolitik der USA und des UK darum geht, soziale Hierarchien zu erhalten oder herzustellen. Normkonformität ist nicht das Ziel. Die Arbeit Wacquants gehört also auch zu den Schriften, die das Thema ,soziale Kontrolle“ ansprechen, dieses Thema aber nicht behandeln.

2.3 Die Thesen, denen zufolge sich die Strafrechts- und Strafverfolgungspolitik immer weniger am Konformitätsziel orientieren, werden in der neueren Kriminalsoziologie großenteils unter dem Sammelbegriff „Punitivitätsthese“ zusammengefasst (vgl. dazu Lautmann und Klimke 2004). Der Begriff „Punitivität“ ist nicht besonders scharf abgegrenzt. Garland, der mit seinem Buch „The Culture of Control“ die Diskussion dieser These stark beeinflusst hat, definiert ihn nicht. Vielleicht könnte man den Begriff mit „Straflust“ übersetzen. Nach Rüdiger Lautmann und Daniela Klimke ist „punitiv“ „,eine bestimmte Art, Strafsanktionen einzusetzen, nämlich mit Härte und Schärfe“" (2004, S. 10). Geht man von dieser Definition aus, so ließe sich relativ leicht feststellen, ob Punitivität gestiegen ist oder nicht. Man müsste nur die 
Entwicklung von Strafurteilen und -gesetzen unter den genannten Gesichtspunkten zählen und auswerten. Ein solches Verfahren aber wäre ziemlich unergiebig. Ergäbe sich nach einer an diesem Verfahren orientierten Untersuchung, dass die Punitivität gestiegen ist, könnte das heißen: Die Kriminellen sind brutaler geworden, ihre Zahl ist gestiegen, neue Arten der Kriminalität hätten sich gebildet usw. Dergleichen wird ja von PunitivitätsbefürworterInnen behauptet. Halten wir uns einmal an die Übersetzung „Straflust“: Die Straflust bliebe ja auf demselben Niveau. Nur gäbe es mehr Anlässe dafür, dass sich diese Lust entäußert. Interessant wären Untersuchungen, deren Ergebnisse zeigten, dass sich die Kriminalitätshäufigkeit nicht verändert habe, die Strafen und Strafgesetze aber schärfer geworden seien.

Im Fall von Strafen hieße das etwa: Man könnte Zeit vergleichende Aktenuntersuchungen durchführen und feststellen, ob bei denselben Kontextbedingungen, innerhalb derer RichterInnen Handlungen von Angeklagten wahrnehmen, unterschiedliche Delikte zugeschrieben und damit unterschiedliche Strafen verhängt werden (vgl. Menzel und Peters 2003, 2008, S. $78 \mathrm{ff}$.).

Kaum zu beantworten ist dagegen die Frage, ob die Strafrechtsentwicklung für oder gegen die Annahme zunehmender Punitivität spricht. Will man auf dieser Ebene zeigen, dass die Punitivität zu- oder abgenommen hat, muss man deutlich machen, dass die Sachverhalte, die als strafwürdig gelten, im Vergleichszeitraum gesellschaftlich als gleich anerkannt worden sind (vgl. Schetsche 1996, S. 14ff.). Eine sehr schwierige Aufgabe.

Christina Schlepper (2014) hat in einer umfangreichen, die deutschen Verhältnisse betreffenden Untersuchung versucht, die Punitivitätsentwicklung auf andere Weise nachzuzeichnen. Sie geht der Frage nach, ,welchen Zielen, Leitprinzipien und Logiken die deutsche Strafgesetzgebung der Spätmoderne folgt“" (58). Von großer Bedeutung sind für sie die Begründungen der Gesetzesänderungen. In Orientierung an Annahmen, die Garland in „The Culture of Control“ formuliert hat, kommt sie auf 13 Begründungsarten (vgl. 60). Ein wesentlicher Teil der Untersuchung besteht in Versuchen zu ermitteln, in welchem Maß die Begründungsarten bei Änderungen des Strafrechts in der Bundesrepublik Deutschland eine Rolle gespielt haben. Die Ergebnisse der Untersuchung Schleppers besagen z.B., dass das Ziel „Resozialisierung“ an Bedeutung verloren, das Ziel „Abschreckung“ dagegen an Bedeutung gewonnen hat (vgl. 156ff.).

Für die Punitivitätsthese sprächen Befunde dieser Art nicht, verstände man sie als Belege für die Annahme, dass eine Verschärfung des Strafrechts auf die Entäußerung von Straflust verwiese. Nicht geprüft wird, ob die BefürworterInnen von Strafverschärfungen von jener Gleichheitsannahme ausgehen, die für diese Annahme grundlegend ist: Dass nämlich die Qualität strafwürdiger Sachverhalte im Untersuchungszeitraum gesellschaftlich als gleich anerkannt wurden. Oder aber: Die BefürworterInnen nehmen keine Veränderung dieser Qualität wahr, meinen aber, dass die bis dahin verhängten Strafen nicht wirksam gewesen seien. Auch dies spräche nicht für die Punitivitätsthese.

Ein weiteres Problem, das sich mit der Formulierung der Punitivitätsthese stellt, ergibt sich aus der Unterschiedlichkeit der Begründungen von Strafrechtsverschärfungen. Es geht zum einen wohl um das ,governing through crime“. Aber sicherlich nicht nur. Das Strafrecht wird auch von ,,atypischen Moralunternehmern“ als Mittel 
verstanden, das geeignet ist, bestimmte Rechtsgüter zu schützen. So ist unter Einfluss feministischer Gruppierungen z. B. das Recht auf sexuelle Selbstbestimmung gestärkt worden. Entsprechende Strafandrohungen wurden verschärft. Vergewaltigung in der Ehe etwa wurde zum Delikt (vgl. Scheerer 1986; vgl. auch Schlepper 2014, S. 197). Offensichtlich wird also das Strafrecht für ganz unterschiedliche und - orientiert man sich an dem eben genannten Beispiel kann man sogar sagen gegensätzliche Ziele eingesetzt: zum Herrschaftserhalt und zum Herrschaftsabbau. Diese Unterschiede werden übersehen, ist man nur an der Frage interessiert, ob Strafen oder das Strafrecht schärfer oder milder geworden sind.

\section{Aktualität}

3.1 Der Begriff ,,soziale Kontrolle“ spielte in der deutschen Soziologie bis in 1950erJahre keine große Rolle (Peters 2012, S. 1256f.). Erst mit Erscheinen des Fischer-Lexikon ,Soziologie“ (1958) wurde er in Fachkreisen einigermaßen bekannt. In diesem 321 Textseiten umfassenden Buch wurden etwa vier - von René König geschriebene - Seiten diesem Begriff gewidmet. Deutlich häufiger verwendet wurde er dann seit der konstruktivistischen Wende der Soziologie abweichenden Verhaltens Ende der 1960er, Anfang der 1970er-Jahre. Seine fast plötzlich entstandene soziologische Popularität war das Nebenprodukt der ebenso plötzlich erfolgenden Verbreitung der Annahme, dass abweichendes Verhalten objektiv nicht vorliege und deswegen nicht als objektiv vorliegendes Verhalten erklärt werden könne. Abweichendes Verhalten sei vielmehr das Produkt von Zuschreibungen und Thematisierungen.

Als „Ursache“ abweichenden Verhaltens galten Instanzen sozialer Kontrolle. Wer abweichendes Verhalten erklären wollte, musste sich diesen Annahmen zufolge mit den Aktivitäten dieser Instanzen befassen. Derartige Vorstellungen büßten dann in den folgenden Jahrzehnten in der deutschen Soziologie an Geltung ein. Das zeigen Ergebnisse einer vergleichenden Untersuchung der Inhalte der ,großen“ deutschen soziologischen Zeitschriften - Berliner Journal für Soziologie, Kölner Zeitschrift für Soziologie und Sozialpsychologie, Soziale Welt, Zeitschrift für Soziologie (vgl. Peters 2018). In der Zeit von 1979 bis 1982 wurden danach in diesen Zeitschriften sechs Artikel zum Thema Devianz veröffentlicht. Die Autoren von zweien dieser Artikel orientierten sich an den Annahmen der „neuen“ Soziologie abweichenden Verhaltens. Beide Autoren erörterten Aktivitäten von Instanzen sozialer Kontrolle. Ein Autor erwähnte die ,neue“ Soziologie abweichenden Verhaltens und auch Instanzen sozialer Kontrolle am Rande. Zwei Autoren orientierten sich nicht an diesen Annahmen, sahen aber die Möglichkeit, ihre Devianzsoziologie mit deren „,neuer“ Variante zu verknüpfen. Ein Autor berücksichtigte die Annahmen der ,neuen“ Soziologie abweichenden Verhaltens nicht. In dem Zeitraum von 2013 bis 2016 wurden in den genannten Zeitschriften 14 Artikel zum Thema Devianz veröffentlicht. Keiner dieser Artikel ließ einen Einfluss der ,neuen“ Soziologie abweichenden Verhaltens erkennen (vgl. Peters 2018, S. 257 ff.).

Im Blick auf den hier thematisierten Begriff ,soziale Kontrolle“ besagt dieses Ergebnis: Ein wichtiger Lieferant für Beiträge zum Thema „soziale Kontrolle“ existiert in der deutschen Soziologie nicht mehr. 
Gibt es Gegenbewegungen? Ein Indikator für die Aktualität des Begriffs ,,soziale Kontrolle" dürfte die Häufigkeit sein, mit der dieser Begriff und die mit ihm angesprochene Thematik in den großen soziologischen Gegenwartsdiagnosen aufgegriffen und erörtert wird. Zu diesen Diagnosen zählen meiner Einschätzung nach Hartmut Rosas „Resonanz“ (2016), Steffen Maus „Das metrische Wir“ (2017) und Andreas Reckwitz’ „Die Gesellschaft der Singularitäten“ (2018). In zweien dieser Arbeiten - denen von Rosa und Reckwitz - spielt der Begriff „soziale Kontrolle“ keine Rolle. Die Autoren kommen bei ihrem Bemühen, die von ihnen für relevant gehaltenen Merkmale der Gegenwartsgesellschaft zu beschreiben und zu erklären, ohne ihn aus. Auch die jeweiligen Register sprechen für diese Annahme. Anders sieht es - wie wir gesehen haben - bei dem Buch von Mau aus. Das Wort ,soziale Kontrolle" wird hier oft verwendet - häufig jedoch in einer Bedeutung, die unserem Begriff „,soziale Kontrolle“ nicht entspricht und deren Verbreitung - wie wir gesehen haben - die soziologische Handhabbarkeit des Begriffs beeinträchtigen würde.

Wenn es um die „großen“ soziologischen Gegenwartsdiagnosen geht, sollte am Rande auch das Buch „Die Sakralität der Person“ von Hans Joas (2011) erwähnt werden, das die deutsche Soziologie stark beschäftigt hat. „Am Rande“, weil es nicht ohne weiteres als „Gegenwartsanalyse“ zu bezeichnen ist. Es geht Joas um - so der Untertitel des Buchs - „Eine neue Genealogie der Menschenrechte“. Der Begriff ,soziale Kontrolle“ taucht in dem Buch - soweit ich sehe - nicht, jedenfalls nicht an prominenter Stelle auf. In unserem thematischen Zusammenhang ist aber bemerkenswert, dass in dem Buch das Thema „Strafe“ ausführlich erörtert wird. Dies allerdings in deutlicher Entfernung zu den in der Soziologie sozialer Kontrolle verbreiteten Fragestellungen. Joas versucht zu zeigen, dass in einem Jahrhunderte währenden Prozess immer nachdrücklicher der Erhalt körperlicher Unversehrtheit gefordert wird. Dies wecke und hemme zugleich den Strafimpuls. „Derselbe Prozeß nämlich, der uns grausame Strafen abzulehnen lehrt, weil wir auch im Verbrecher den Menschen sehen und diesen respektieren, macht uns zugleich empfindlicher gegenüber der Grausamkeit des Verbrechens“, schreibt Joas (2011, S. 97). Man könnte diese Einschätzung als Ansatz zur Beschreibung moderner Kontrolltendenzen verstehen: Die Neigung, Devianzen ohne Opfer, die ja niemanden verletzen, immer weniger und Devianzen mit Opfern, insbesondere Gewalt gegen Menschen, immer heftiger zu bestrafen (vgl. Peters 2013, S. 55 ff.). Dies wäre eine soziologisch bemerkenswerte Interpretation der Erörterungen Joas'. Sie geht aber an seinem Interesse - darauf weist schon der Titel seines Buchs hin - vorbei.

3.2 Sollte die Soziologie auf den hier empfohlenen enggefassten Begriff ,,soziale Kontrolle“ verzichten? Vieles spricht dafür, dass die gegenwärtige deutsche Soziologie wenig dagegen einzuwenden hätte. Und Verzichtsvorschläge wurden ja auch schon gemacht (vgl. Steinert 1995, S. 82; Cremer-Schäfer 1995, S. 89; vgl. auch French et al. 2018, S. 66).

Es wäre wohl ein Verlust für die Soziologie. Würde das, was unter sozialer Kontrolle verstanden wird, eingehen in die Bedeutungen der Begriffe Institutionalisierung, Erziehung, Sozialisation, soziale Zwänge, Sozialdisziplinierung usw., verlören soziale Vorgänge an soziologischer - und wohl auch politischer - Beachtung, die 
besonders intensiv in das Leben der von ihnen Betroffenen eingreifen. Es sind vor allem zwei Merkmale des Begriffs, die entschieden für seinen Erhalt und für die Hervorhebung seiner soziologischen Bedeutung sprechen: Zum einen lenkt er die soziologische Aufmerksamkeit auf Fehlentwicklungen der Kontrollpraxis, die auch bei Anerkennung manifester Sanktionsziele als unmenschlich gelten. Zu erwähnen sind hier Untersuchungen, die der stets aktuellen Frage nachgehen, in welchem Maß Sanktionen das Konformitätsziel erreichen. Unsere Zeitschrift hat sich mit dieser Frage ausführlich befasst (vgl. etwa Kury und Scherr 2013). Zum anderen birgt der eng gefasste Begriff ,,soziale Kontrolle“ das Potenzial für die Schärfung des Bewusstseins, dass Aktivitäten von Instanzen sozialer Kontrolle als Reaktionen allenfalls halb verstanden werden. Der Begriff kann angesichts der Definitionsmacht von Instanzen sozialer Kontrolle Anstoß für die Annahme sein, dass sich Devianz durch die Aktivität von sozialer Kontrolle erst herstellt - und zwar uno actu. Mit dem Schrumpfen des Interesses der deutschen Soziologie am Begriff „,soziale Kontrolle“ vergrößert sich die Gefahr, dass dieses Potenzial nicht genutzt wird.

Funding Open Access funding enabled and organized by Projekt DEAL.

Open Access Dieser Artikel wird unter der Creative Commons Namensnennung 4.0 International Lizenz veröffentlicht, welche die Nutzung, Vervielfältigung, Bearbeitung, Verbreitung und Wiedergabe in jeglichem Medium und Format erlaubt, sofern Sie den/die ursprünglichen Autor(en) und die Quelle ordnungsgemäß nennen, einen Link zur Creative Commons Lizenz beifügen und angeben, ob Änderungen vorgenommen wurden.

Die in diesem Artikel enthaltenen Bilder und sonstiges Drittmaterial unterliegen ebenfalls der genannten Creative Commons Lizenz, sofern sich aus der Abbildungslegende nichts anderes ergibt. Sofern das betreffende Material nicht unter der genannten Creative Commons Lizenz steht und die betreffende Handlung nicht nach gesetzlichen Vorschriften erlaubt ist, ist für die oben aufgeführten Weiterverwendungen des Materials die Einwilligung des jeweiligen Rechteinhabers einzuholen.

Weitere Details zur Lizenz entnehmen Sie bitte der Lizenzinformation auf http://creativecommons.org/ licenses/by/4.0/deed.de.

\section{Literatur}

Berger, L. Peter, und Thomas Luckmann. 1969. Die gesellschaftliche Konstruktion der Wirklichkeit. Eine Theorie der Wissenssoziologie. Frankfurt a. M: S. Fischer.

Christie, Nils. 2005. Wieviel Kriminalität braucht die Gesellschaft? München: C. H. Beck.

Cremer-Schäfer, Helga. 1995. Aussortieren und Einsortieren. Zur Funktion der Strafe bei der Verwaltung der sozialen Ausschließung. Kriminologisches Journal 27(2):89-119.

Edelman, Murray. 1988. Die Erzeugung und Verwendung sozialer Probleme. Journal für Sozialforschung 28(2):175-192.

French, Martin, et al. 2018. Epidemics, pandemics, and outbreaks. In The cambridge handbook of social problems, Bd. 2, Hrsg. A. Javier Trevino, 59-78. Cambridge, New York: Cambridge University Press.

Garland, David. 2001. The culture of control. Crime and social order in contemporary society. Oxford: Oxford Press.

Joas, Hans. 2011. Die Sakralität der Person. Eine neue Genealogie der Menschenrechte. Berlin: Suhrkamp. König, René. 1958. Soziale Kontrolle. In Fischer Lexikon „Soziologie“, Hrsg. René König, 253-257. Frankfurt a. M.: Fischer Bücherei.

Kury, Helmut, und Albert Scherr. Hg. 2013. Zur (Nicht-)Wirkung von Sanktionen. Immer härtere Strafen - immer weniger Kriminalität? Soziale Probleme, 24(1). 
Lautmann, Rüdiger, und Daniela Klimke. 2004. Punitivität als Schlüsselbegriff für eine Kritische Kriminologie. In Punitivität, Hrsg. Rüdiger Lautmann, Daniela Klimke, und Fritz Sack. Kriminologisches Journal 8:9-29.

Lemert, Edwin M. 1951. Social pathology. A systematic approach to the theory of sociopathic behavior. New York: McGraw-Hill.

Lucke, Doris Mathilde. 2014. Kontrolle, soziale. In Wörterbuch der Soziologie, Hrsg. Günter Endruweit, Gisela Trommsdorff, und Nicole Burzan, 245-248. Konstanz: UTB.

Mau, Steffen. 2017. Das metrische Wir. Über die Quantifizierung des Sozialen. Berlin: Suhrkamp.

Menzel, Birgit. 2019. Soziale Kontrolle. Kriminologisches Journal 51(1):72-78.

Menzel, Birgit, und Helge Peters. 2003. Sexuelle Gewalt. Eine definitionstheoretische Untersuchung. Konstanz: UVK.

Menzel, Birgit, und Helge Peters. 2008. Problemgeneratoren. Bestrafung von Sexualtätern mit ,,ungeregelter Lebensführung“. In Exklusion der Marktwirtschaft, Hrsg. Daniela Klimke, 77-86. Wiesbaden: VS.

Peters, Helge. 2002. Soziale Probleme und soziale Kontrolle. Wiesbaden: Westdeutscher Verlag.

Peters, Helge. 2009. Devianz und soziale Kontrolle. Eine Einführung in die Soziologie abweichenden Verhaltens. Weinheim, München: Juventa.

Peters, Helge. 2012. Soziale Kontrolle. In Handbuch soziale Probleme, Bd. 2, Hrsg. Günter Albrecht, Axel Groenemeyer, 1255-1285. Wiesbaden: Springer VS.

Peters, Helge. 2013. Politiken und Konjunkturen des Strafens. In Hilfe! Strafe! Reflexionen zu einem Spannungsverhältnis professionellen Handelns, Hrsg. Peter Rieker, et al., 42-59. Weinheim, Basel: Beltz, Juventa.

Peters, Helge. 2018. Elitäre Sekte. Zur Rezeption der interaktionstheoretisch orientierten Devianz- und Kriminalsoziologie. Kriminologisches Journal 50(4):257-273.

Reckwitz, Andreas. 2018. Die Gesellschaft der Singularitäten. Berlin: Suhrkamp.

Rosa, Hartmut. 2016. Resonanz. Eine Soziologie der Weltbeziehung. Berlin: Suhrkamp.

Sack, Fritz. 2008. Die deutsche Kriminologie im Lichte des Werkes von D. Garland. In Exklusion in der Marktgesellschaft, Hrsg. Daniela Klimke, 301-322. Wiesbaden: VS.

Scheerer, Sebastian. 1986. Atypische Moralunternehmer. Kriminologisches Journal 1:133-156.

Schetsche, Michael. 1996. Die Karriere sozialer Probleme. Soziologische Einführung. München, Wien: Oldenbourg.

Schlepper, Christina. 2014. Strafgesetzgebung in der Spätmoderne. Wiesbaden: Springer VS.

Schmitt-Leonardy, Charlotte. 2019. Verständigung als gelungene Strategie im Umgang mit Informalisierungstendenzen im Strafverfahren. Kriminologisches Journal 51(3):212-232.

Simon, Jonathan. 1997. Gewalt, Rache und Risiko. Die Todesstrafe im neoliberalen Staat. In Soziologie der Gewalt, Hrsg. Trutz von Trotha. Kölner Zeitschrift für Soziologie und Sozialpsychologie 37:279-301.

Singelnstein, Tobias, und Peer Stolle. 2012. Die Sicherheitsgesellschaft. Soziale Kontrolle im 21. Jahrhundert. Wiesbaden: VS.

Steinert, Heinz. 1995. Soziale Ausschließung - Das richtige Thema zur richtigen Zeit. Kriminologisches Journal 27(2):82-88.

Wacquant, Loic. 2009. Bestrafen der Armen. Zur neoliberalen Regierung der sozialen Unsicherheit. Opladen, Farmington Hills: Barbara Budrich.

Weber, Max. 1964. Wirtschaft und Gesellschaft, erster Halbband. Köln, Berlin: Kiepenheuer \& Witsch. 